\title{
D2D communication for spectral efficiency improvement and interference reduction: A survey
}

\author{
Abdullah H. Alquhali, Mardeni Roslee, Mohamad Y. Alias, Khalid S. Mohamed \\ Centre for Wireless Technology (CWT), Faculty of Engineering, Multimedia University, Malay sia
}

\begin{tabular}{|c|c|}
\hline Article Info & ABSTRACT \\
\hline Article history: & Device-to-device (D2D) communication system plays an extremely \\
\hline Received Oct 21, 2019 & $\begin{array}{l}\text { important role in fulfilling the demands of fourth generation }(4 \mathrm{G}) \text { and fifth } \\
\text { generation }(5 \mathrm{G}) \text { technologies. } 4 \mathrm{G} \text { technology cannot meet high data }\end{array}$ \\
\hline Revised Dec 28, 2019 & demands. The D2D communication system is believed to provide major \\
\hline Accepted Feb 14, 2020 & $\begin{array}{l}\text { improvements in resource usage, energy efficiency, and overall throughput, } \\
\text { which are the main demands for the } 5 \mathrm{G} \text { network. Some of the main }\end{array}$ \\
\hline Keywords: & $\begin{array}{l}\text { issues in the } \mathrm{D} 2 \mathrm{D} \text { communication system involve spectrum efficiency } \\
\text { and interference. Although many studies have been conducted on spectrum }\end{array}$ \\
\hline $5 \mathrm{G}$ & efficiency improvement and interference reduction, the issues still remain. \\
\hline Device-to-device & communication system and to develop a D2D scheme with efficient spectrum \\
\hline In-band communication & utilization and interference reduction. Many survey papers have been \\
\hline LTE-A & published on these issues, but the fundamental concepts behind the D2D \\
\hline Spectrum efficiency & $\begin{array}{l}\text { communication system require investigation. In this study, we investigated } \\
\text { and analyzed the fundamental concepts behind the D2D communication } \\
\text { system to develop an efficient D2D resource allocation scheme with } \\
\text { reduced interference. }\end{array}$ \\
\hline
\end{tabular}

This is an open access article under the CC BY-SA license.

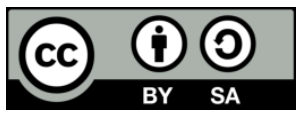

Corresponding Author:

Mardeni Roslee,

Centre for Wireless Technology (CWT),

Faculty of Engineering, Multimedia University,

Persiaran Multimedia, 63100 Cyberjaya, Selangor, Malaysia.

Email: mardeni.roslee@mmu.edu.my

\section{INTRODUCTION}

Telecommunication firms are struggling to fulfil the total demand of mobile users. New mobile applications, such as proximity-aware services, are launched in the market every day, in which most of them have extremely intense data usage. Although the fourth generation $(4 \mathrm{G})$ cellular technologies have extremely efficient performance in physical and media access control layers, they cannot meet the data demands of mobile users and are lagging behind because of high traffic and extensive load on base stations (BSs) [1-3]. The global mobile traffic has increased by a thousand times in this decade as predicted by the Mobile Communication Enablers for the 2020 Information Society (METIS) [4, 5]. The increasing number of Internet of Things (IoT) connected devices, such as tablets, mobile phones, video sharing, social networking, and online gaming, requires huge data demands. Therefore, the upcoming generation cellular technologies should be developed to fulfil such demands. Several technologies are under development, where device-to-device (D2D) communication exhibits huge potential.

D2D communication describes the direct transmission of data packets between user equipment (UE). The conventional design of cellular communication can only manage small data rate services, although UE are in the vicinity of each other. Some challenges, such as spectral efficiency, energy efficiency, 
data interference reduction, and transmission delay, occur when dealing with such scenarios because of high data rate requirements and the existence of end users exist in proximity. Many spectral utilization prototypes are proposed by researchers. However, they still induce harmful interference among nodes and cellular users (CUs). This study investigates different spectrum utilization approaches and finds a method to enhance spectrum utilization for reducing undesirable interferences that degrade the overall network performance.

In D2D communication, the spectrum can be shared between D2D and CUs using two methods, namely, inband and outband communication [4-7], where the resource sharing mechanism is implemented using underlay and overlay communication [8-10]. Resource management is controlled using controlled and autonomous approaches, which are also known as centralized and distributed control sche mes [11-15] as shown in Figure 1. The rest of this paper is organized as follows. Section 2 describes the general concepts, advantages, and disadvantages of D2D communication. Section 3 discusses the literature on the basic designs of $\mathrm{D} 2 \mathrm{D}$ communication and the structures in which a D2D system can be developed. Section 4 provides the conclusions, general comments, and recommendations on the D2D system designs proposed on the basis of the literature. Table 1 provides the list of acronyms used in this study.

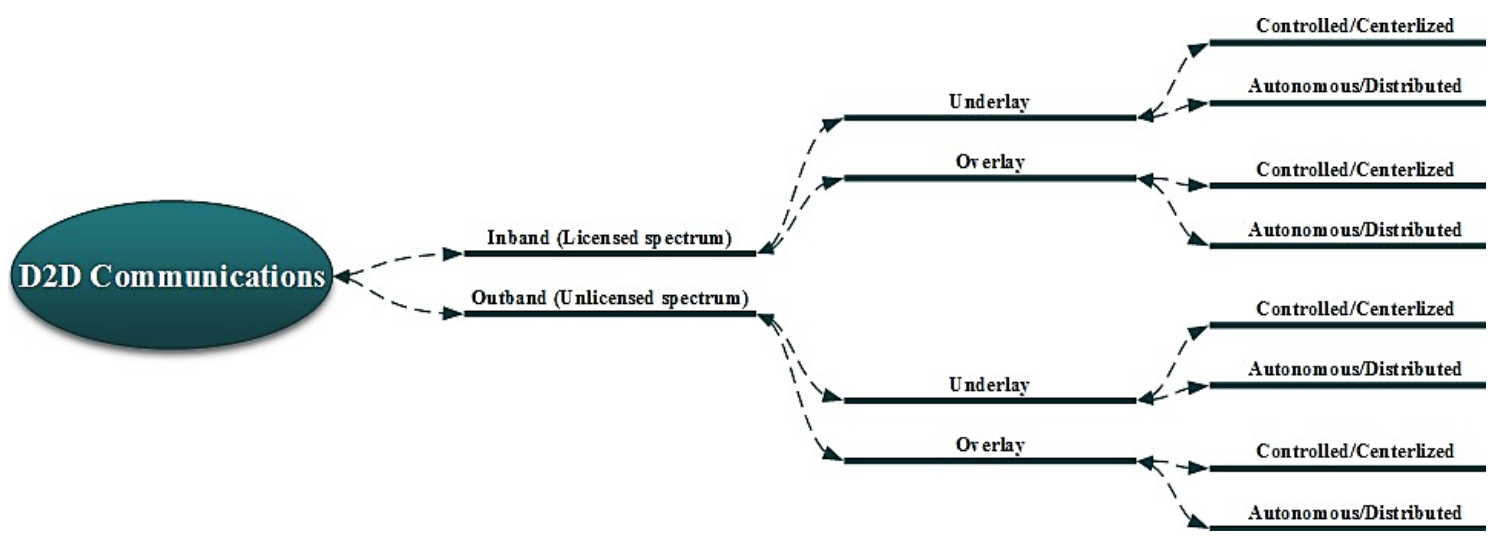

Figure 1. Device-to-device communication architecture

Table 1 . List of acronyms

\begin{tabular}{ll}
\hline Acronyms & Full form \\
\hline D2D & Device-to-Device \\
IoT & Internet of things \\
4G & Fourth Generation \\
MAC & Media Access Control \\
ProSe & Proximity Services \\
METIS & $\begin{array}{l}\text { Mobile and Wireless communications Enablers for } \\
\text { the T wenty-twenty Information Society }\end{array}$ \\
QoS & Quality of Service \\
5G & Fifth Generation \\
LTE-U & Long Term Evolution in Unlicensed spectrum \\
UL & Up-link \\
DL & Down-link \\
LTE-A & Long Term Evolution Advance \\
eNB & Evolved NodeB \\
HetNets & Heterogeneous Networks \\
UEs & User Equipments \\
CU & Cellular User \\
OFDM & Orthogonal Frequency Division Multiplexing \\
BS & Base Station \\
CSI & Channel State Information \\
mm-wave & Millimeter-wave \\
\hline &
\end{tabular}

\section{GENERAL CONCEPTS OF D2D COMMUNICATION SYSTEM}

Although many studies have been conducted on D2D communication, some complexities are encountered in understanding the terms used in general D2D communication systems. Thus, this study first focuses on understanding each term used in D2D communication taxonomy. Table 2 summarizes the merits and disadvantages of D2D communication. 


\subsection{Inband communication}

The literature in this category has been widely investigated and proposed the use of the cellular licensed spectrum for cellular and D2D links. Inband communication is selected to acquire high and efficient utilization for the spectrum because it enables high control on the cellular licensed spectrum [16-18]. Researchers have extensively investigated several inband communication schemes and their ability to mitigate interferences by controlling the licensed spectrum [2]. High traffic and congestion-related issues may arise when huge number of communication establishment requests and demands for data rates are received, such as public safety, video games, and content sharing [19]. Therefore, inband communication is effective in improving spectral efficiency [20] because the BS can control the entire communication system [21].

\subsection{Outband communication}

Unlicensed spectrum is utilized by customers during outbound communication. The fundamental advantage of this spectrum is that is free of charge [22]. This communication technique is suitable in uncrowded areas, whereas it becomes extremely challenging in high-traffic areas because of interference when the number of users increases [23]. Outband communication mainly aims to accommodate adequate data rate in local area networks, such as industries, public areas, and personal area networks [3]. This technique also aims to minimize the interference caused by D2D to CUs [24]. Unlicensed spectra are widely used in industrial, scientific, and medical communication systems that use personal area network technologies and adhoc networks. Such spectra are mostly used for short-range communication systems, such as Wi-Fi Direct and Bluetooth [25].

\subsection{Underlay communication}

This is the first resource sharing mechanism, in this resource sharing mechanism, the communication spectrum is shared between the cellular and D2D users, both of these users can communicate at the same time using the same radio resources [26]. D2D users and cellular users can use a single channel utilizing mixed time slots [24]. Therefore, sometimes this communication system is also known as the mixed-mode communication [16]. This system can cause interference when it is improperly managed because it allows multiple users to work together, indicating that the network supports many users utilizing the same number of resources [27]. The system spectrum efficiency is improved when the number of resources remains the same with the increase in the number of users [28], as shown in Figure 2.

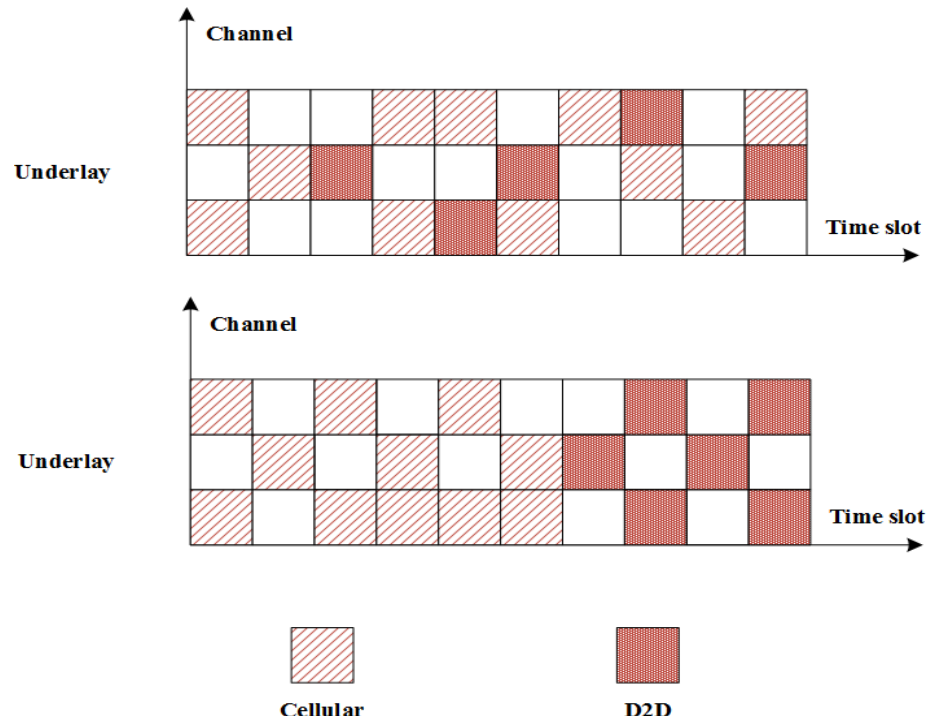

Figure 2. Resource sharing mechanism (reproduced from [3])

\subsection{Overlay communication}

In this communication, the spectrum between D2D and CUs is separated, where a portion of the spectrum is used by D2D users, and the other portion is dedicated for CUs. In this way, a fraction of the channels is used by the former, whereas the remaining channels are utilized by the latter [29]. In other words, a single channel is shared between D2D and CUs at different time slots [30]. The initial time slots can be 
given to CUs, whereas the last time slots can be given to D2D users. In this communication system, D2D users occupy the empty cellular spectrum for communication [31]. In this communication system, interference is mitigated but the spectrum efficiency is reduced because D2D users are served at the expense of CUs [32], as shown in Figure2.

\subsection{Controlled or centralized mechanism}

In this mechanism, the cellular network operator controls the as signment of radio resources, indicating that the BS controls the operation of UE and is responsible for all the tasks related to device communication systems, such as resource allocation, power control, and mode selection [33]. The QoS of each D2D UE is controlled by the BS in such a way that it provides the facility for optimizing the spectrum resource usage and reducing the interference [34]. This system is simple because the BS controls the overall system and has all the information to reduce the conflicts caused by different devices, and the dynamic system is efficiently handled [35]. This control system can cause the failure of the entire communication system with the failure of a single [36].

\subsection{Autonomous or distributed mechanism}

In this mechanism, individual UE are responsible for the D2D communication system. Hence, the communication channel access and interference control functions are implemented at the user side [37]. The UE would require the CSI of the complete network by fetching the local information of the remaining UE [38]. This type of control system can be used without considering the nature of individual UE, thereby resulting in fast decision making and quick variation to dynamic system environments. Individual nodes are autonomous in making decisions, thereby resulting in rapid adaptation of the dynamic system and decision making [5]. However, the UE can possibly make decisions on its own because the system is autonomous. Therefore, multiple decisions can cause the divergence of the overall joint decision [39]. This condition complicates the communication system because of the large and complex signal processing requirement at the user end. Therefore, the system should be efficiently optimized when making decisions in a dynamic systemto obtain solutions with optimal efficiency [40], as shown in Figure 3.

Table 2. Comparison between the D2D mechanisms

\begin{tabular}{|c|c|c|c|}
\hline Mechanism & Description & Advantages & Disadvantages \\
\hline Inband & $\begin{array}{l}\text { This mechanismis } \\
\text { widely investigated } \\
\text { and uses the cellular } \\
\text { licensed spectrum } \\
\text { for cellular and D2D } \\
\text { links. Therefore, } \\
\text { inband } \\
\text { communication is } \\
\text { used to acquire } \\
\text { highly efficient } \\
\text { utilization of the } \\
\text { spectrum and high } \\
\text { control of the } \\
\text { licensed cellular } \\
\text { licensed spectrum }\end{array}$ & $\begin{array}{l}\text { - CUs efficiently utilize D2D underlay } \\
\text { because of the spatial diversity } \\
\text { - CUs Clear regulation of QoS because the BS } \\
\text { controls the licensed mobile spectrum } \\
\text { - Any CU can use in- band D2D } \\
\text { communications because the cell interface is } \\
\text { typically not allowed at out band frequencies } \\
\text { - Free of charge } \\
\text { - Dedicating cellular resources to the D2D } \\
\text { spectrum is not required in underlay inband } \\
\text { because the CU can reuse, the UL frequency } \\
\text { compared with the overlay inband } \\
\text { - Interference can be controlled and no } \\
\text { interplatform is required } \\
\text { - Does not require more than one interface for } \\
\text { devices } \\
\text { - Improve the performance in underlay }\end{array}$ & $\begin{array}{l}- \text { The interference between the CU } \\
\text { and D2D users is extremely difficult } \\
\text { in underlay communication } \\
\text { compared with } \\
\text { overlaycommunication. } \\
- \text { The spectral efficiency in overlay } \\
\text { communication is reduced because } \\
\text { of the waste of cell resources } \\
\text { - Cannot simultaneously transmit (UL) } \\
\text { D2D and cellular spectrum } \\
- \text { High comput ation complexity } \\
\text { of scheduler resource allocation } \\
\text { and power control optimization } \\
- \text { High cost }\end{array}$ \\
\hline Outband & $\begin{array}{l}\text { Unlicensed } \\
\text { spectrum is } \\
\text { exploited by the } \\
\text { user. Therefore, } \\
\text { outband D2D } \\
\text { communication is } \\
\text { used to mitigate the } \\
\text { interference bet ween } \\
\text { D2D and cellular } \\
\text { link. }\end{array}$ & $\begin{array}{l}\text { - The unlicensed outband spectrum is used is } \\
\text { to mitigate the interference bet ween D2D } \\
\text { and CUs } \\
\text { - Free of charge } \\
\text { - No need to dedicate resources of CUs to } \\
\text { D2D users (e.g., overlay) } \\
\text { - Less comput ational complexity to scheduler } \\
\text { resource allocation approaches and power } \\
\text { control optimization } \\
\text { - D2D and cellular users can Simultaneously } \\
\text { transmit data }\end{array}$ & $\begin{array}{l}\text { - Interference cannot be controlled } \\
\text { because the spectrum is unlicensed } \\
\text { - Only cellular devices with two radio } \\
\text { interfaces (e.g., LTE and Wi-Fi) can } \\
\text { use outband D2D communication } \\
\text { - Efficient power management } \\
\text { between two wireless interfaces is } \\
\text { crucial. Otherwise, the power } \\
\text { consumption of the device increases } \\
\text { - Packets (headers) should be } \\
\text { decoded and encoded } \\
\text { be-cause the protocols used by } \\
\text { different radio interfaces are different } \\
\text { - Uncontrolled natural interference } \\
\text { (unlicensed spectrum) }\end{array}$ \\
\hline
\end{tabular}


Table 2. Comparison between the D2D mechanisms (continue)

\begin{tabular}{|c|c|c|c|}
\hline Mechanism & Description & Advantages & Disadvantages \\
\hline $\begin{array}{l}\text { Centralized } \\
\text { /Con-trolled }\end{array}$ & $\begin{array}{l}\text { The key node applies } \\
\text { bandwidth to D2D and } \\
\text { B2D connections. The } \\
\text { BS uses the channel } \\
\text { quality indicator of all } \\
\text { B2D and D2D links to } \\
\text { assign the spectrum to } \\
\text { the B2D and D2D } \\
\text { connetions using } \\
\text { centralized techniques. } \\
\text { Consequently, the BS } \\
\text { is responsible for all } \\
\text { computer and } \\
\text { management tasks }\end{array}$ & $\begin{array}{l}\text { Low interference between the devices } \\
\text { Efficient handling of the dynamic system } \\
\text { Improves the reliability and performance }\end{array}$ & $\begin{array}{l}\text { - } \quad \text { The entire system can fail because } \\
\text { of single point failure. } \\
\text { - } \quad \text { The overload of the BScan cause } \\
\text { limited } \\
-\quad \text { Poor availability } \\
-\quad \text { Lack of failure tolerance }\end{array}$ \\
\hline $\begin{array}{l}\text { Distributed } \\
\text { /Au- } \\
\text { tonomous }\end{array}$ & $\begin{array}{l}\text { Individual UE are } \\
\text { responsible for } \\
\text { allocating the } \\
\text { spectrum for D2D } \\
\text { communication. } \\
\text { Therefore, the UE } \\
\text { acquire the CSI of the } \\
\text { entire network by } \\
\text { exchanging their local } \\
\text { information with other } \\
\text { UE. }\end{array}$ & $\begin{array}{l}\text { Fast adaptation of the dynamic system } \\
\text { Can be used in the absence of coverage } \\
\text { Reduces the overhead of the cellular network }\end{array}$ & $\begin{array}{l}\text { - User equipment can make a decision } \\
\text { on their own } \\
\text { - Multiple decisions may cause } \\
\text { the divergence of the overall joint } \\
\text { decision } \\
\text { - High computational complexity }\end{array}$ \\
\hline
\end{tabular}

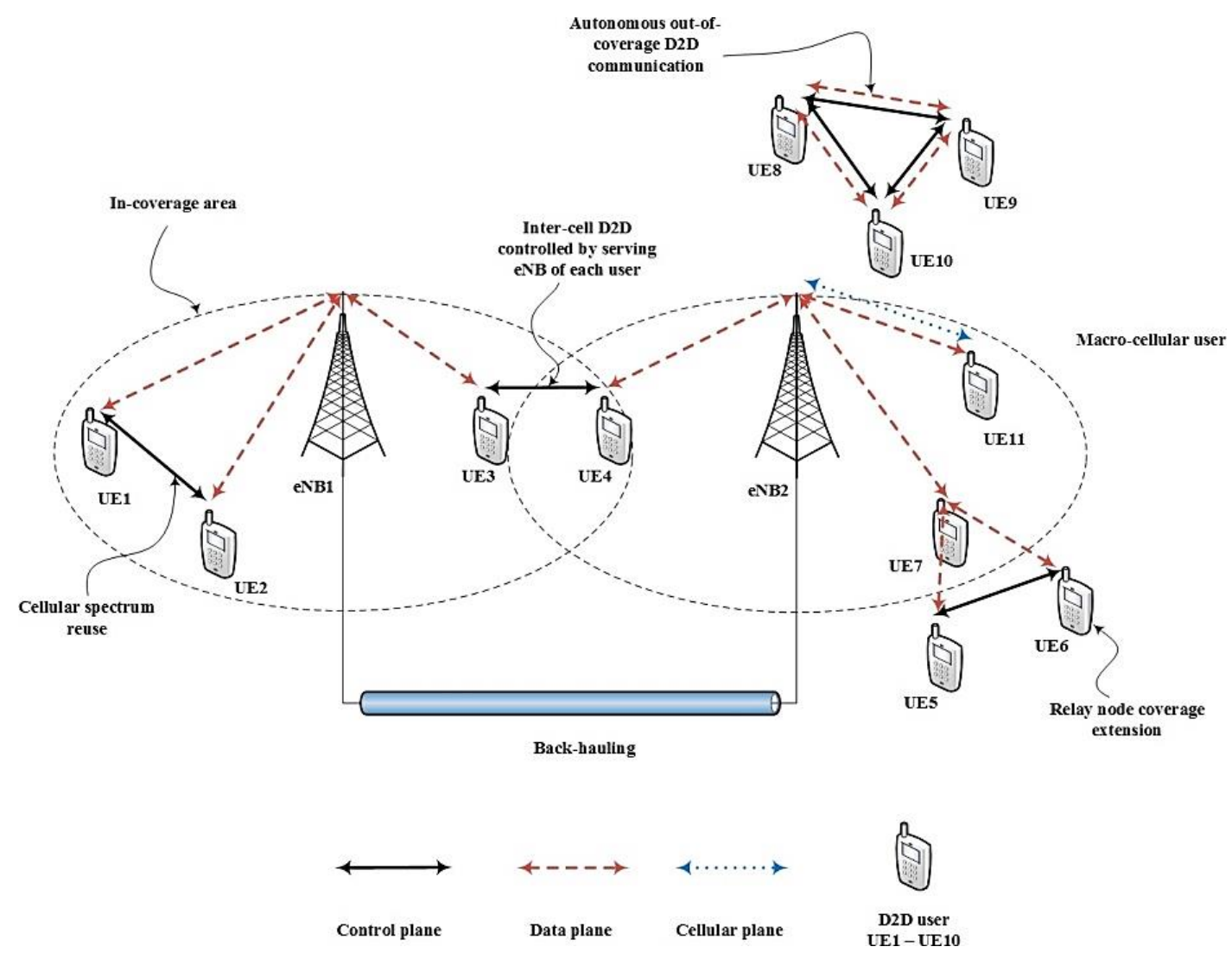

Figure 3. A D2D enabled cellular network [40]

\section{D2D COMMUNICATION SYSTEM DESIGNS}

Algorithm structures for D2D communication system have been developed using different approaches. This study presents the general approaches that can be utilized to develop D2D prototypes. This study reviews the algorithms and approaches used for the development of D2D prototypes. A D2D algorithm can be designed using eight fundamental approaches, as shown in Figure 4. However, this study 
only focuses on the inband communication system approaches, as shown in Figures 4-7. The authors in [41] a random resource allocation scheme to mitigate interference and improve the spectrum efficiency. In this method, available D2D devices can reuse any CU channel resources. The interference of UL devices can be controlled using this method without increasing the distance between the users. However, efficient utilization of available frequency bands cannot be obtained in this D2D scheme. The authors in [7, 42] presented a resource allocation scheme that divides the entire cell area into two non-overlapping regions without sectionalizing the cell.

The system throughput is improved by reusing more than one channel resources of CUs. However, the network model is not analyzed for densely allocated users. In [43], a post resource allocation scheme was introduced, where the reuse scenario of frequency is determined on the basis of the interference caused by D2D equipment. The drawback of this system is that an omnidirectional antenna was considered, thereby causing noise in the system. In [44], a Q-learning resource allocation algorithm was developed for LTE-U networks with DL and UL decoupling. The design of this algorithm is shown in Figure 4. They formulated a spectrum allocation problem on the basis of a game theoretic model to incorporate user association, spectrum allocation, and load balancing.

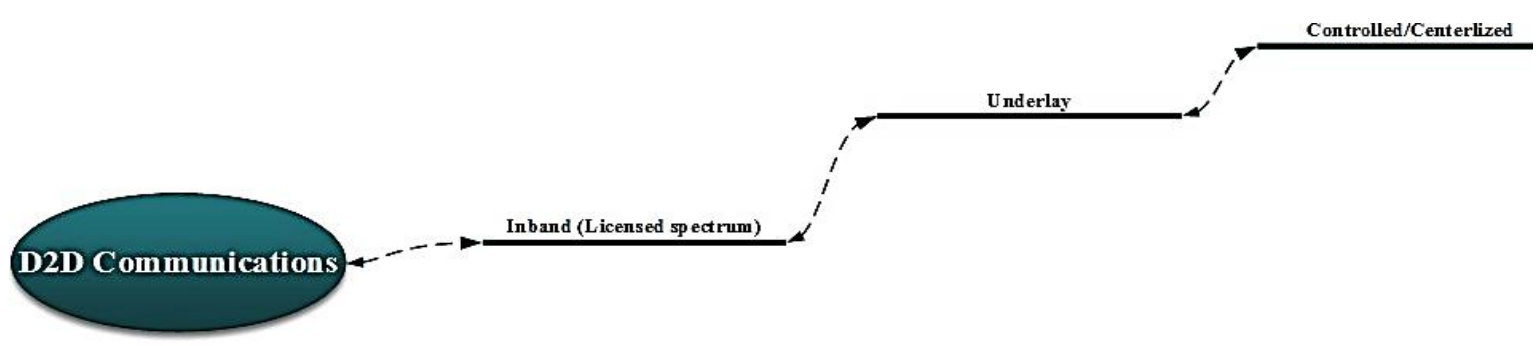

Figure 4. Configuration-1

The design of this algorithm is shown in Figure 5. The main advantage of this method is that the BS can allocate resources although the information about the network is limited. The authors in [10], proposed a D2D communication approach using two steps, where the resource allocation is analyzed in this first step, and the interference reduction problem is designed in the second step. However, the QoS was not analyzed. In [9], a fractional frequency reuse-based method was presented to re-utilize the subsisting cellular channel resource for reducing the traffic load on the eNB to decrease the end-to-end delay and improve the system performance and spectral efficiency. Although these objectives are achieved to some extent, cellular and D2D users still experience some interferences. The authors integrated a distance-based resource allocation system with a fractional frequency reuse mechanism to reduce the interference. The proposed design experiences less interference and exhibits better system performance than the system proposed by [41, 42]. The design of the above mentioned D2D mechanisms is based on Figure 4.

The above mentioned algorithms have some limitations, such as cannot achieve efficient utilization of available frequency bands [41]. The system in [42] do not analyze the densely allocated users, the system in [43] leads to noise and interference, and the system in [10] ignores QoS analysis. The interference between D2D and CUs is ignored in the system of [9] and has several interferences. All systems cause congestion to the BS.

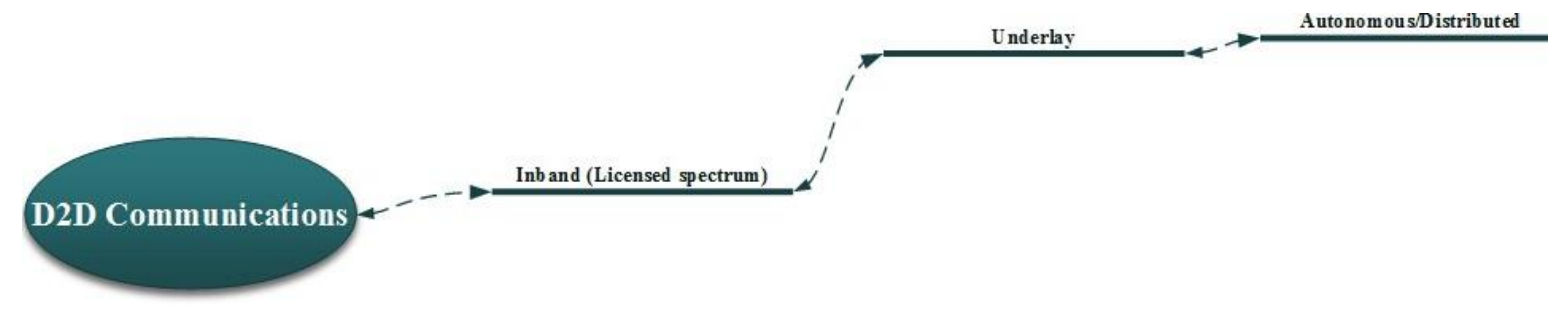

Figure 5. Configuration-2

As shown in Figure 4, inband communication is applied in many D2D schemes to improve the spectrum efficiency. A resource allocation scheme based on Q-learning and configuration 2 is proposed 
using a single-tier network. The resource is shared between the cellular and D2D users to maximize resource throughput. This type of solution can be extremely efficient in solving the resource allocation problems in $5 \mathrm{G}$ networks [45]. The authors in [46] presented a Q-learning-based resource allocation scheme using a two-tier network. The configuration 2 framework aids systems in generating strategies by sharing information for selecting the resource blocks and calculating the input from activities in the context of QoS specifications. The main disadvantage of this algorithm is that the network model considers only one macro cell and only one user is connected to a single femtocell, which is impractical. In [47], an autonomous resource allocation scheme using an autonomous Q-learning algorithm was develop to improve the spectrum efficiency and control the interference in D2D-enabled multi-tier HetNets. This algorithm aims to autonomously reduce the load on the BS and the spectrum using the devices and to increase the throughput of D2D users while maintaining the QoS requirements and outage ratio of CUs.

The learning methodology is to use the interactions with the environment to adapt the dynamic network conditions. The optimality of the throughput indicates that the signal-to-noise ratio, cellular outage ratio, computation time, and spectral efficiency of this technique is better than the distance-constrained resource sharing scheme [11] that mainly focuses on selecting the CU equipment for a D2D link to share its resources. The authors formulated the resource block, channel communication, modulation, and coding scheme as one resource allocation problem using joint resource allocation and linked algorithm to obtain an effective solution [48]. Similar techniques requiring network assistance [8, 49] have been proposed in the past decade. The resource block can be automatically selected by D2D users using a network-assisted interference mitigation scheme with the same configuration [50]. The D2D systems designed based on the configuration in Figure 5 lack spectral efficiency and are impractical. The scheme proposed by [46] is impractical because it only uses one macrocell and only one user is connected to a single femtocell. The remaining systems proposed by [11,47-49] either cause synchronization loss, unreliability, require network as sistance, or increase complexity.

Millimeter waves can be integrated with the D2D technology to improve the spectrum efficiency and the performance of 5G. The author in [14] developed a system based on Figure 6 to reduce the complexity of interference avoidance in high-traffic areas. The inband resource selectively switches to overlay mode under high traffic, where the resource assigned using quadrative programming is designed and formulated to increase the system capacity using a heuristic algorithm. This system exhibits good performance under high traffic and improves the spectrum efficiency because of the selective operation mode [14]. Other researchers have presented other mechanisms where the D2D communication systems can be considered a disruptive technology for 5G. D2D communication can be centralized and distributed, that is, devices can be controlled by the BS or can be autonomously switched [12, 13, 51]. In [13] proposed a system based on configuration 3 to mitigate the interference and improve the resource efficiency through an efficient channel assignment design. This scheme is compared with the full reuse channel assignment algorithm, where every D2D equipment is used to completely share the channel resource, indicating that this scheme performs better in terms of spectrum reuse, systemblocking, and energy efficiency.

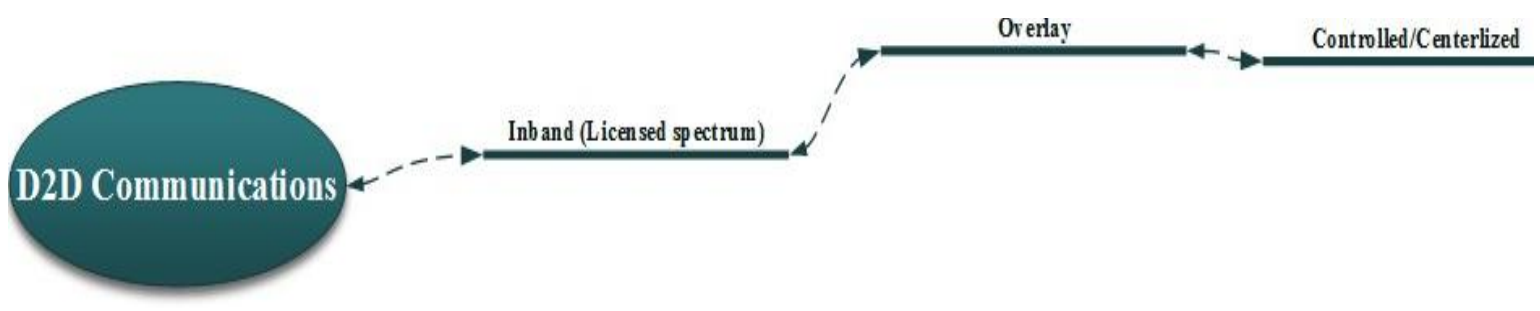

Figure 6. Configuration-3

In [52], a D2D resource allocation algorithm based on the location of vehicles and vehicle-to-vehicle broadcasting was introduced. This algorithm is based on separate locations and applies configuration 3 . The results showed that this algorithm performs well in terms of resource usage, transmission accuracy, and time delay, indicating that the resource utilization efficiency is improved. Another advantage of this system is that the packet error rate is controlled to an acceptable range. However, the system has high load on equipment.

The main advantages of these systems are that they mitigate the interference and improve the spectral efficiency using mode selection. However, these systems should be improved in terms of reliability and consistency. In [4] considered a D2D scenario where D2D users can communicate bidirectionally. This communication system is designed on the basis of Figure 7. D2D users could 
communicate with the cellular BS and UE. In this system, the relay selection from the D2D users is considered because multiple pairs of D2D users can exist. This system is effective in improving the performance ofD2D and CUs. The relay selection is effective in increasing the sum rate of the cellular networks. The author in [53] developed a method for spectrum allocations based on configuration 4, where the D2D equipment decides about its transmission mode by measuring the activity on the D2D spectrum and using a carrier sensing threshold. In this way, the performance of D2D communication can be improved, thereby reducing the interference. The signalling is reduced between the BSs and the users because the system is autonomous. The signalling between the overhead D2D users and their home BSs is completely eliminated, and the high bandwidth signals of CUs are allocated to D2D. The drawback of this system is that the objective function is optimized for D2D users because the rate of CUs is only regarded as the optimization constraint.

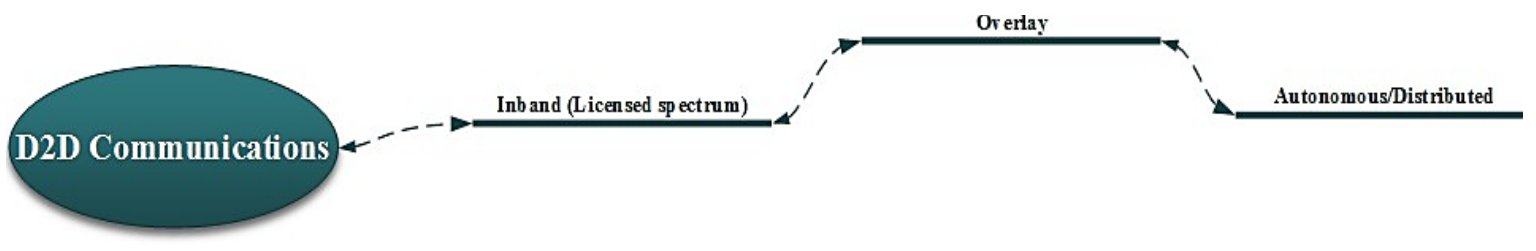

Figure 7. Configuration-4

\section{SUMMARY}

A D2D communication system can be designed on the basis of some fundamental concepts, such as spectrum authorization type, spectrum allocation mechanism, and system control mechanism. Inband communication is widely used in the literature because it reduces the interference between the devices. Outbound communication is widely used in industrial applications. However, it results in many interferences caused by unlicensed authorization policies. Although the underlay spectrum mechanism increases the spectrum efficiency, the communication spectrum shared between the cellular and D2D equipment results in interference. The overlay spectrum mechanism is used in different spectrum channel slots because it reduces the interference at the expense of the spectrum efficiency. The centralized control system is simple, optimizes the spectrum resource usage, and reduces the interference. A single connection problem can cause failure of the entire system and lead to BS overload issues. In the distributed control method, communication can be established in the absence of coverage, thereby resulting in the rapid adaptation of dynamic system and decision making. However, multiple decisions can cause the divergence of the overall joint decision, thereby complicating the communication system and requiring complex signal processing at the user end.

All the mechanisms have some advantages and disadvantages. Researchers have developed mixed mode solutions, such as centralized communication system, to achieve improved flexibility where the BS can be automatically switched to a distributed system until it reaches affordable load conditions. Similarly, the underlay communication system can be switched to overlay communication system when the traffic exceeds the limit, and the system can be automatically changed to the underlying system with the reduction in traffic. D2D communication systems should be improved.

\section{REFERENCES}

[1] H. Tullberg et al., "Metis research and standardization: A path towards a 5G sy stem," Glob. Works.,pp.577-582, 2014.

[2] A. Asadi, Q. Wang, and V. Mancuso, "A survey on device-to-device communication in cellular networks," IEEE Communications Surveys \& Tutorials, vol. 16, no. 4, pp. 1801-1819, 2014.

[3] M. Ahmad et al., "Resource management in D2D communication: An optimization perspective," Journal of Network and Computer Applications, vol. 93, pp. 51-75, 2017.

[4] Y. Pei and Y. C. Liang, "Resource allocation for device-to-device communications overlaying two-way cellular networks," IEEE Transactions on Wireless Communications, vol. 12, no. 7, pp. 3611-3621, 2013.

[5] A. H. Sakr and E. Hossain, "Cognitive and energy harvesting-based D2D communication in cellular net-works: Stochastic geometry modeling and analy sis," IEEE Transactions on Commu., vol. 63, no. 5, pp. 1867-1880, 2015.

[6] Y. Pan et al., "Content offloading via D2D communications based on user interests and sharing willingness," IEEE International Conference on Communications, pp. 1-6, 2017.

[7] Z. Zhang, R. Q. Hu, and Y. Qian, "D2D communication underlay in uplink cellular networks with distance based power control," IEEE International Conference on Communications, pp. 1-6, 2006.

[8] T. Peng, Q. Lu, H. Wang, S. Xu, and W. Wang, "Interference avoidance mechanisms in the hybrid cellular and device-to-device systems," IEEE 20th Int. Symp. on Pers., Indoor and Mob. Radio Comm., pp. 617-621, 2009. 
[9] D. D. Ningombam and S. Shin, "Non-orthogonal resource sharing optimization for D2D communication in LTE-A cellular networks: A fractional frequency reuse-based approach," Electronics, vol. 7, no. 10, pp. 1-20, 2018.

[10] G. Katsinis, E. E. Tsiropoulou, and S. Pap avassiliou, "On the performance evaluation of distributed resource block and power allocation in D2D-enabled multi-cell networks," 14th ACM Symp. on Perf. Ev. of Wire. Ad Hoc, pp. 77-84,2017.

[11] H. Wang and X. Chu, "Distance-constrained resource-sharing criteria for device-to-device communications underlaying cellular networks," Electronics Letters, vol. 48, no. 9, pp. 528-530, 2012.

[12] F. Boccardi, R. W. Heath, A. Lozano, T. L. Marzetta, and P. Popovski, "Five disruptive technology directions for 5G," IEEE Communications Magazine, vol. 52, no. 2, pp. 74-80, 2014.

[13] J. Qiao et al., "Enabling device-to-device communications in millimeter-wave 5G cellular networks," IEEE Communications Magazine, vol. 53, no. 1, pp. 209-215, 2015.

[14] G. D. Swetha and G. R. Murthy, "Selective overlay mode operation for D2D communication in dense 5G cellular networks," IEEE Symposium on Computers and Communications, pp. 704-709, 2017.

[15] T. Adiono, S. F. Anindya, S. Fuada, and M. Y. Fathany, "Curtain control systems development on mesh wireless network of the smart home," Bulletin of Electrical Engineering and Informatics, vol. 7, no. 4, pp. 615-625, 2018.

[16] H. Tang and Z. Ding, "Mixed mode transmission and resource allocation for D2D communication," IEEE Transactions on Wireless Communications, vol. 15, no. 1, pp. 162-175, 2015.

[17] A. Alhammadi et al., "Auto tuning self-optimization algorithm for mobility management in LTE-A and 5G hetnets,"IEEE Access, vol. 8, pp. 294-304, 2019.

[18] S. Su and S. Wang, "A simple monitoring network system of wireless sensor network," Bulletin of Electrical Engineering and Informatics, vol. 1, no. 4, pp. 251-254, 2012.

[19] Y. Shen, C. Jiang, T. Q. S. Quek, and Y. Ren, "Device-to-device-assisted communications in cellularnetworks: An energy efficient ap proach in downlink video sharing scenario," Trans. on Wire. Comm., vol. 15, no. 2, pp. 1575-1587, 2015.

[20] K. S. Mohamed, M. Y. Alias, and M. Roslee, "Interference avoidance using TDM A-beamforming in location aware small cell systems," Applied Sciences, vol. 9, no. 23, pp. 1-11, 2019.

[21] G. Araniti, A. Raschellà, A. Orsino, L. Militano, and M. Conduluci, "Device-to-device communications over 5G systems: Standardization, challenges and open issues," 5G Mobile Communications, Springer, pp.337-360, 2017.

[22] K. Doppler, M. Rinne, C. Wijting, C. B. Ribeiro, and K. Hugl, "Device-to-device communication as an underlay to LTE-advanced networks," IEEE Communications Magazine, vol. 47, no. 12, pp. 42-49, 2009.

[23] S. Hakola, T. Chen,J. Lehtomäki, and T.Koskela, "Device-to-device (D2D) communication in cellularnet work-performance analy sis of optimum and practical communication mode selection," Wire. Comm. and Net. Conf., pp. 1-6, 2010.

[24] X. Lin, J. G. Andrews, and A. Ghosh, "Spectrum sharing for device-to-device communication in cellular networks,"IEEE Transactions on Wireless Communications, vol. 13, no. 12, pp. 6727-6740, 2014.

[25] G. Fodor et al., "Design aspects of network assisted device-to-device communications," IEEE Communications Magazine, vol. 50, no. 3, pp. 170-177, 2012.

[26] C. Ma et al., "Interference exploitation in D2D-enabled cellular networks: A secrecy perspective," IEEE Transactions on Communications, vol. 63, no. 1, pp. 229-242, 2014.

[27] W. Cheng, X. Zhang, and H. Zhang, "Optimal power allocation with statistical qos provisioning for D2D and cellular communications over underlay ing wireless networks," J. on Selec. Areas in Comm., vol. 34, no. 1, pp. 151-162, 2015.

[28] A. Afzal, S. A. R. Zaidi, D. McLernon, and M. Ghogho, "On the analysis of device-to-device overlaid cellular networks in the up link under 3GPP propagation model," Wireless Comm. and Net. Conf., pp. 1-6, 2016.

[29] K. Zhu and E. Hossain, "Joint mode selection and spectrum partitioning for device-to-device communication: A dynamic stackelberg game," Transactions on Wireless Comm., vol. 14, no. 3, pp. 1406-1420, 2014.

[30] Q. Ye, M. Al-Shalash, and C. Caramanis, "Resource optimization in device-to-device cellular systems using time-frequency hopping," IEEE Transactions on Wireless Communications, vol. 13, no. 10, pp. 5467-5480, 2014.

[31] E. Ahmed, I. Yaqoob, A. Gani, M. Imran, and M. Guizani, "Social-aware resource allocation and optimization for D2D communication," IEEE Wireless Communications, vol. 24, no. 3, pp. 122-129, 2017.

[32] Y. Zhang et al., "Social network aware device-to-device communication in wireless networks," IEEE Transactions on Wireless Communications, vol. 14, no. 1, pp. 177-190, 2014.

[33] L. Lei, Z. Zhong, C. Lin, and X. Shen, "Operator controlled device-to-device communications in LTE-advanced networks," IEEE Wireless Communications, vol. 19, no. 3, pp.96-104, 2012.

[34] Y. Li, Z. Wang, D. Jin, and S. Chen, "Optimal mobile content downloading in device-to-device communication underlaying cellular networks," Transactions on Wireless Comm., vol. 13, no. 7, pp. 3596-3608, 2014.

[35] H. Min, W. Seo, J. Lee, S. Park, and D. Hong, "Reliability improvement using receive mode selection in the deviceto-device up link period underlaying cellular networks," Trans. on Wire. Comm., vol. 10, no. 2, pp. 413-418, 2010.

[36] E. Datsika, A. Antonopoulos, N. Zorba, and C. Verikoukis, "Green cooperative device-to-device communication: A social-aware perspective," IEEE Access, vol. 4, pp. 3697-3707, 2016.

[37] R. I. Ansari et al., "5G D2D networks: Techniques, challenges, and future prospects," IEEE Systems Journal, vol. 12, no. 4, pp. 3970-3984, 2017.

[38] F. Wang et al., "Energy-aware resource allocation for device-to-device underlay communication," IEEE international conference on communications, pp.6076-6080, 2013.

[39] A. Pyattaev et al., "Network-assisted D2D communications: Implementing a technology prototype for cellular traffic offloading," IEEE Wireless Communications and Networking Conference, pp. 3266-3271, 2014.

[40] G. A. Safdar, M. Ur-Rehman, M. Muhammad, M. A. Imran, and R. Tafazolli, "Interference mitigation in D2D communication underlaying LTE-A network," IEEE Access, vol. 4, pp. 7967-7987, 2016.

[41] Q. Duong, Y. Shin, and O. Shin., "Distance-based resource allocation scheme for device-to-device communications underlaying cellular networks," AEU Int. J. Electro. Commu., vol. 69, no. 10, pp. 1437-1444, 2015.

[42] Z. Zhang, R. Q. Hu, Y. Qian, and A. Papathanassiou, "D2D communication underlay in uplink cellular networks with fractional power control and fractional frequency reuse," Global Comm. Conf., pp. 1-7, 2015. 
[43] N. P. Kuruvatti and H. D. Schotten, "Post-resource sharing power allocation in cellular networks to coexist with D2D underlay," 7th International Conference on the Network of the Future, pp. 1-3, 2016.

[44] Y. Hu, R. MacKenzie, and M. Hao, "Expected q-learning for self-organizing resource allocation in LTE-U with downlink-uplink decoupling," European Wireless 2017; 23th European Wireless Conference, pp. 1-6, 2017.

[45] Y. Luo, Z. Shi, X. Zhou, Q. liu, and Q. Yi, "Dynamic resource allocations based on q-learning for D2D communication in cellular networks," 11th Int. Comp. Conf on Wav. Actiev Med. Tech. and Inf. Process., pp. 385-388, 2014.

[46] I. AlQerm and B. Shihada, "A cooperative online learning scheme for resource allocation in 5G systems," IEEE International Conference on Communications, pp. 1-7, 2016.

[47] K. Zia et al., "A distributed multi-agent RL-based autonomous spectrum allocation scheme in D2D enabled multi-tier hetnets," IEEE Access, vol. 7, pp.6733-6745, 2019.

[48] S. Rostami, K. Arshad, and P. Rapajic, "A joint resource allocation and link adaptation algorithm with carrier aggregation for 5G LTE-advanced network," Int. Conf. on Telecommunications, pp. 102-106, 2015.

[49] M. Zulhasnine, C. Huang, and A. Srinivasan., "Efficient resource allocation for device-to-device communication underlaying LTE network," IEEE Int. Conf. on Wireless and Mobile Comp., Net and Commu., pp. 368-375, 2010.

[50] A-H. Tsai, L-C. Wang, J-H. Huang, and T-M. Lin, "Intelligent resource management for device-to-device (D2D) communications in heterogeneous networks," The 15 th Int. Symp. on Wire. Personal Multi. Comm., pp. 75-79, 2012.

[51] M. N. Tehrani, M. Usyal, and H. Yanikomeroglu, "Device-to-device communication in 5G cellular networks, challenges, solutions, and future directions," IEEE Communications Magazine, vol. 52, no. 5, pp. 86-92, 2014.

[52] X. Zhang, Y. Shang, X. Li, and J. Fang "Research on overlay D2D resource scheduling algorithms for V2V broadcast service," IEEE 84th Vehicular Technology Conference, pp. 1-5, 2016.

[53] B. Cho, K. Koufos, and R. Ja"ntti, "Spectrum allocation and mode selection for overlay D2D using carrier sensing threshold," 9th Int. Conf. on Cognitive Radio Oriented Wireless Networks and Communications, pp. 26-31, 2014.

\section{BIOGRAPHIES OF AUTHORS}

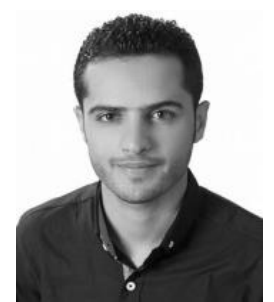

Abdullah H. Alquhali received the B.Eng degree in electrical and communications engineering from Sana'a University in 2012 and Master of Engineering in telecommunications from Multimedia Uni- versity (Malaysia) in 2018. He is currently doing his Ph.D. degree in Efficient Spectrum Utilization Algorithm Based on Distance of 4G and 5G D2D Wireless Technologies. His current research inter- ests include 4G and 5G, resource allocation, spectrum efficiency and Device-to-Device communica- tions, and next generation cellular networks.

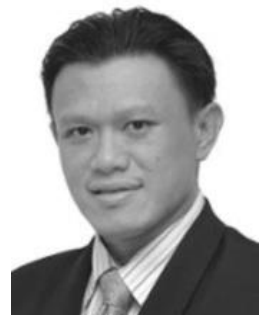

Mardeni Roslee is a registered Chartered Engineer with the Engineering Council United Kingdom, and Member with The Institution of Engineering and Technology (IET), United Kingdom. As a Chartered Engineer, he bring a diversified range of engineering experience in design, development and engineering management. His experiences include the consultation, professional institution and academic sectors. He is a Senior Member of IEEE and senior member of IACSIT. His current research interests are wireless mobile communication and radar communication system.

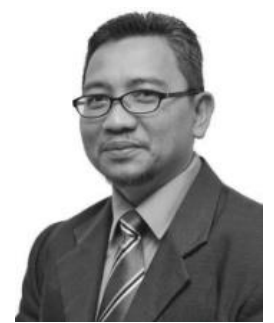

Mohamad Y. Alias obtained the bachelor of science in engineering (Electrical Engineering) degree from the University of Michigan, Ann Arbor, in May 1998. He then received his Ph.D. degree in December 2004 from the School of ECS, University of Southampton in the United Kingdom. He is currently a Professor at the Faculty of Engineering, Multimedia University in Malaysia. His research interests cover the field of wireless communications especially in OFDM, multiple antenna system, multiuser detection, genetic algorithms in communications, multimedia applications, and visible light communications

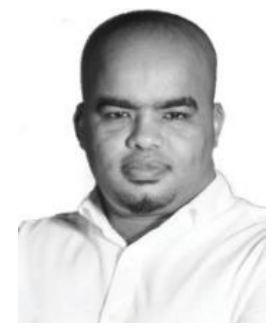

Khalid S. Mohamad received his B.Eng. and M.Eng. in Telecommunication from Future Uni- versity, Sudan in 2011 and Multimedia University, Malaysia in 2014, respectively. He is currently pursuing his Ph.D. in Telecommunication in Multimedia University, Malaysia. His research interests include Cellular communication, LTE, 5G, Interference Management in Heterogeneous Networks 